\title{
MGMR3D, a semi-analytic code for the obtaining the radio foot- print from the shower currents
}

\author{
Olaf Scholten ${ }^{1,2, \star}$, Gia Trinh ${ }^{1}$, Krijn D. de Vries $^{2}$, and Brian Hare ${ }^{1}$ \\ ${ }^{1}$ University of Groningen, KVI Center for Advanced Radiation Technology, 9747 AA Groningen, The Nether- \\ lands \\ ${ }^{2}$ Vrije Universiteit Brussel, Dienst ELEM, IIHE, Pleinlaan 2, 1050 Brussels, Belgium
}

\begin{abstract}
MGMR3D is a code that calculates the complete radio footprint, i.e. intensity, polarization and pulse shapes, for a parametrized shower current density using a semianalytic approach. The non-Monte-Carlo code is fast (typically 10 seconds for a complete footprint) and thus can be used in a chi-square optimization calculation of atmospheric electric fields or to optimize antenna geometry.
\end{abstract}

In response to request from the community we have build a code that is fast, predictive, and calculates the complete radio footprint from first principles using the 3-dimensional structure of an extensive air shower. As such it can be used in a chi-square optimization code to determine the current structure as function of height in the atmosphere as is needed for determining electric fields in (thunder-)clouds. It can also be used for any application where it is needed to calculate the footprint of many showers to -as an example- optimize an antenna layout. The code can also be used to investigate the sensitivity of the radio footprint to shower parameters. The supplementary material contains a somewhat extended version of this contribution.

Since the code calculates the footprint from first principles once the currents are specified, the results include Cherenkov effects due to a finite refractivity of air. To optimize the cpu time we use conveniently chosen parametrizations for the shower current in the transverse and longitudinal (charge excess) directions. The code is written such that it is relatively easy to adapt these parametrizations. Calculations with MGMR3D [1] are compared with those of CoREAS [2]. Discrepancies with the microscopic calculation are an indication that the chosen parametrization does not reflect all aspects of the air shower physics.

In Fig. 1 the footprint is compared for a non-trivial current geometry as may occur during a thunderstorm. Between the heights of 3 and $8 \mathrm{~km}$ the electric field $(50 \mathrm{kV} / \mathrm{m})$ is oriented in the opposite direction as from the $3 \mathrm{~km}$ down ward $(15 \mathrm{kV} / \mathrm{m})$. The field is furthermore oriented perpendicular to the $\mathbf{v} \times \mathbf{B}$ direction. This results in an intensity pattern that shows a clear ring-like structure and $Q / I=-1$ implying a polarization along the $\mathbf{v} \times(\mathbf{v} \times \mathbf{B})$ direction. The explanation for the ring-like intensity pattern in given in Fig. 2. Since the direction of the induced transverse currents changes at $3 \mathrm{~km}$ height, the radio emission of the two heights will interfere destructively for an observer close to the shower core (observer 1). For an observer at a larger distance from the core (observer 2) the radiation from the top layer will arrive later than the radiation from the bottom layer. Depending on the timing, i.e. the distance from the shower axis, the two contributions may interfere constructively

\footnotetext{
^e-mail: Scholten@KVI.nl
} 

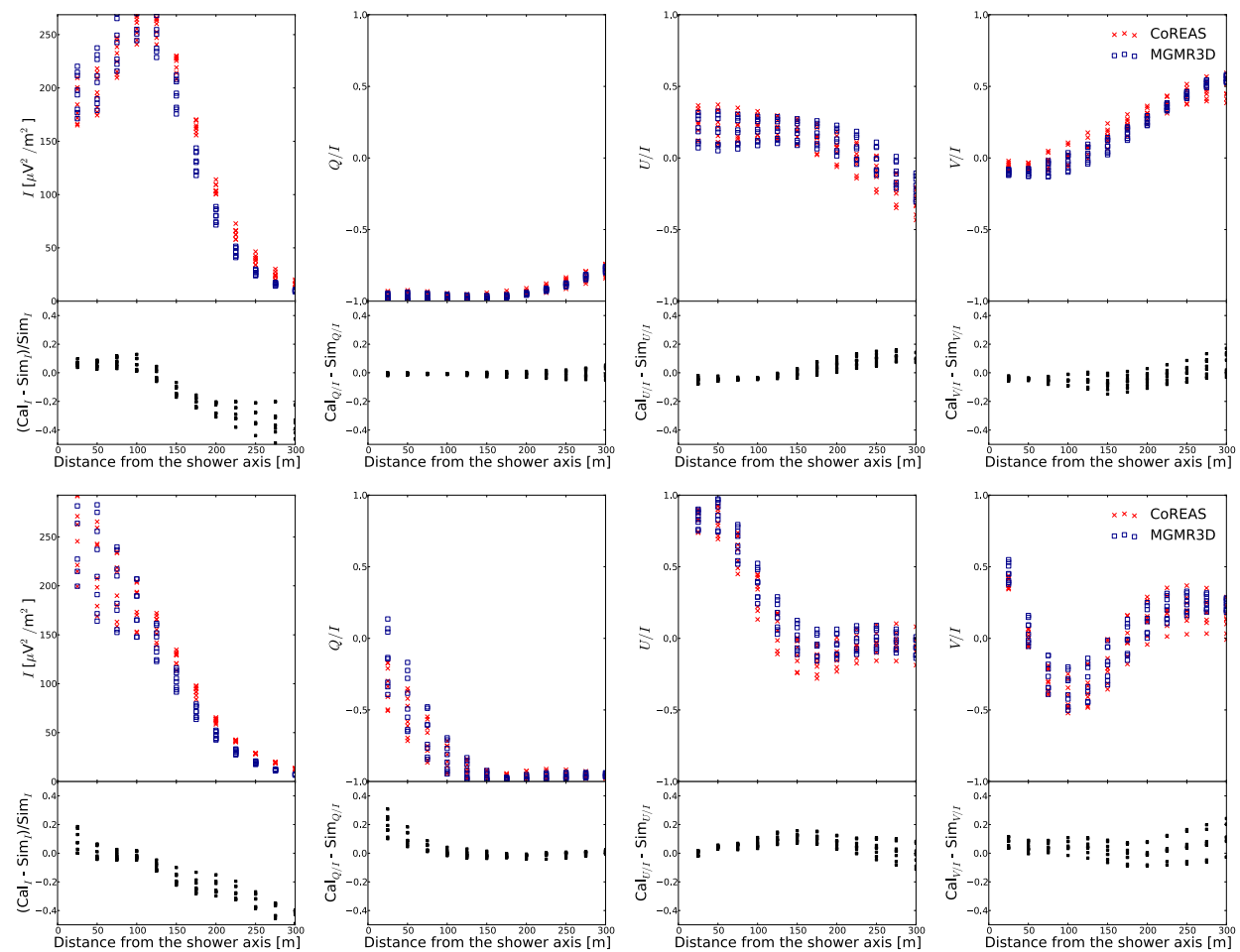

Figure 1. Comparison of the results for a dual and triple-layered atmospheric electric field.
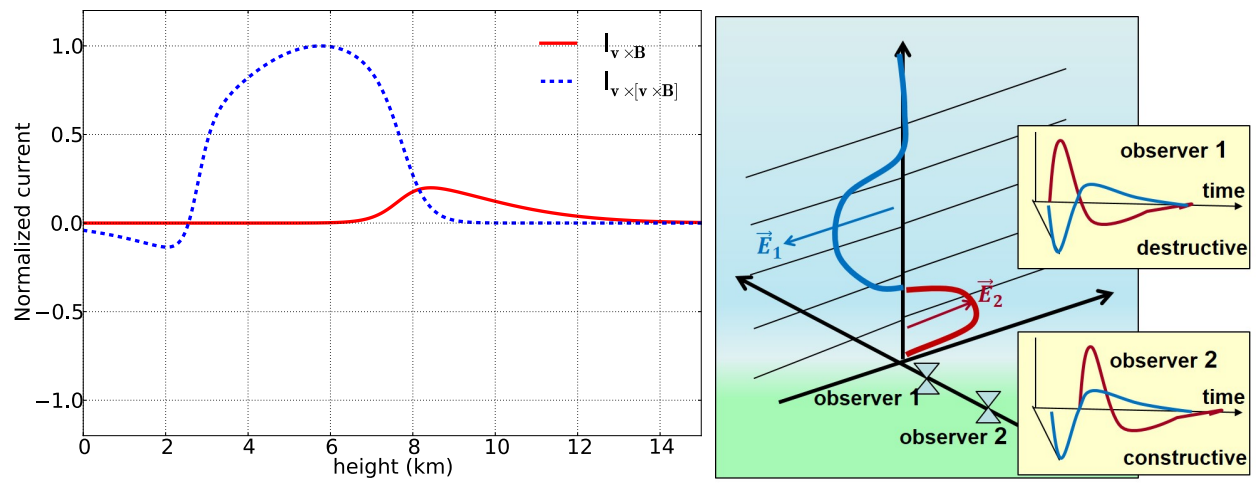

Figure 2. The ring structure interpreted, see text.

thus creating a ring-like intensity pattern. The linear polarization in the $\mathbf{v} \times(\mathbf{v} \times \mathbf{B})$ direction is due to the fact that the currents induced by the electric field is far stronger than the geomagnetic current.

The main difference of the field configuration used in the example of Fig. 1, bottom panel, from that of the previous example is that the field in the lowest layer is now taken along the $\mathbf{v} \times \mathbf{B}$ direction. 

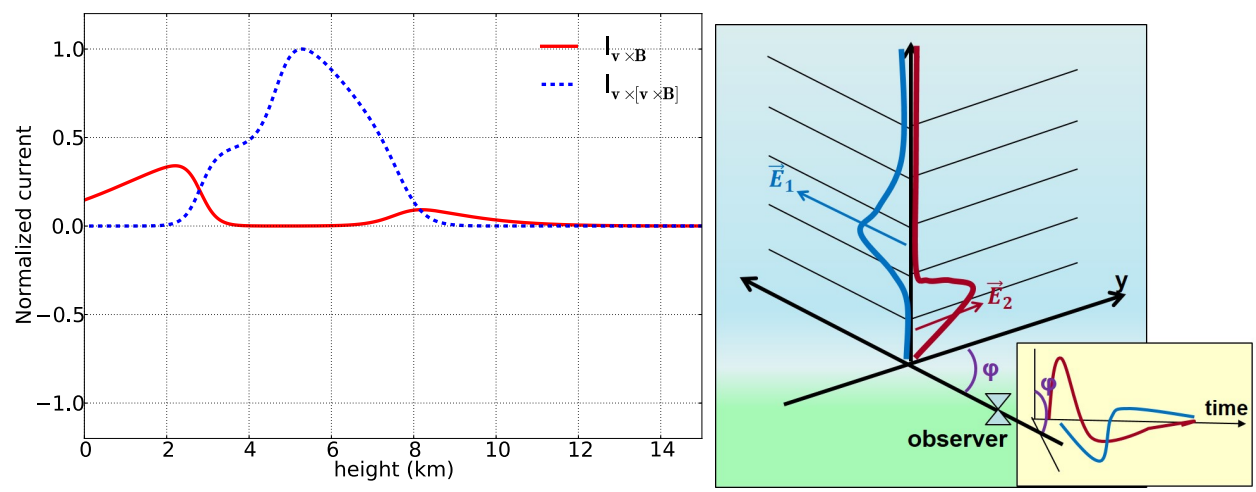

Figure 3. The circular polarization interpreted, see text.

Thus these two contributions do not interfere any more. However now the $\mathbf{v} \times(\mathbf{v} \times \mathbf{B})$ polarized pulse and the $\mathbf{v} \times \mathbf{B}$ polarized pulse arrive simultaneously for an observer near the shower core resulting in a pulse polarized in the $45^{\circ}$ direction. This results in the Stokes parameter $U / I=+1$. The two emissions will receive a relative delay that depends on the distance to the shower axis as shown in Fig. 3. Depending on the delay the polarization during the pulse rotates left or right, expressed by Stokes $V / I$ that changes sign. This intricate dependence of the polarization observables on the shower currents is a clear indication that for an accurate determination of the currents it is imperative to include all Stokes parameters in the analysis [3, 4].

Even though the physics and current structures are complicated it is clear from Fig. 1 that the MGMR3D and CoREAS results lie rather close to each other. This is shows that MGMR3D, in spite of its parametrized shower structure, captures the essence. It is thus possible to use MGMR3D in a chi-square fitting approach to extract from the measured radio footprint the structure of the currents in the atmosphere [5-8].

\section{References}

[1] O. Scholten, T. N. G. Trinh, K. D. de Vries, and B. M. Hare (2018), Phys. Rev. D 97, 023005 (2018)

[2] T. Huege, M. Ludwig, and C. James, AIP Conf. Proc. 1535, 128 (2012)

[3] P. Schellart, et al., J. Cosm. and Astrop. Phys. 10, 014 (2014); arXiv:1406.1355

[4] O. Scholten, et al., Phys. Rev. D 94, 103010 (2016), arXiv:1611.00758

[5] P. Schellart et al., Phys. Rev. Lett 114, 165001 (2015), arXiv:1504.05742

[6] G. Trinh, O. Scholten, et al., Phys. Rev. D 93, 023003 (2016),

arXiv:1511.03045.[7] T.N.G.Trinh, et al., Phys. Rev. D 95, 083004 (2017),

arXiv: 1703.06008

[8] Gia Trinh et al., Contribution to these proceedings 